\title{
On the stability of long-range sound propagation through a structured ocean
}

\author{
Michael A. Wolfson and Steven Tomsovic \\ Department of Physics, Washington State University, Pullman, WA 99164-2814.
}

(October 25, 2018)

\section{PACS Numbers: 43.30.Cq, 43.30.Ft, 43.30.Pc}

Several acoustic experiments show a surprising degree of stability in wave fronts propagating over multi-megameter ranges through the ocean's sound channel despite the presence of random-like, sound speed fluctuations. Previous works have pointed out the existence of chaos in simplified ray models incorporating structure inspired by the true ocean environment. A "predictability horizon" has been introduced beyond which stable wave fronts cease to exist and point-wise, detailed comparisons between even the most sophisticated models and experiment may be limited for fundamental reasons. We find, by applying one of the simplified models, that for finite ranges, the fluctuations of the ray stabilities are very broad and consistent with lognormal densities. A fraction of the rays retain a much more stable character than the typical ray. This may be one of several possible mechanisms leading to greater than anticipated sound field stability. The lognormal ray stability density may underlie the recent, experimentally determined, lognormal density of wave field intensities [J. Acoust. Soc. Am. 105, 3202-3218 (1999)].

\section{INTRODUCTION}

There is a great deal of experimental and theoretical interest in long-range, low-frequency acoustic pulse propagation through the deep ocean's sound channel. It has been investigated as a problem of wave propagation in random media (WPRM) [1,2], and as a basis for tomography [3,4]. Recent results from the Acoustic Engineering Test (AET) as part of the Acoustic Thermometry of Ocean Climate (ATOC) project can be found in Colosi et al. [5] and Worcester et al. \#6. One of the main challenges in analyzing and understanding long range acoustic propagation is in dealing with difficulties arising from the ocean environment's tendency to generate multiple, weak, small-angle (forward) scattering [7]. At sufficiently long ranges of propagation, the multiple scattering should effectively randomize an acoustic pulse so that it is very difficult to deduce much information. However, several long range experiments have found a great deal of stability in the earlier portions of the received wave fronts in spite of the fluctuations inherent in the ocean environment 8,9, 9, In addition, it has been found that wave field intensity fluctuations at long range are consistent with a lognormal density [5] which would be reminiscent of earlier work in optics on WPRM 10, ex- cept that this earlier work was for the short range (weak focusing) regime.

In the past 10-15 years, simplified models inspired by the ocean environment have been shown to possess chaotic ray limits 11 13. Essentially simultaneously, there has been enormous progress in the understanding of chaotic systems [14]. Some of the most familiar emerging concepts are simpler for bounded systems and are not easily applicable to open, scattering systems as we have here. However, there is an important tool which does straightforwardly generalize for our purposes, the stability analysis of the rays. Stability matrices can be constructed as a function of range for each ray. Their properties, such as the stability exponents, reveal the basic character of the rays, and are at the foundation of the findings reported in this paper.

There are several intriguing questions that arise from comparing the theoretical results to date regarding chaotic acoustic ray dynamics in the ocean and the high amount of stability observed in the data. The most general question concerns how an acoustic pulse - which at multi-megameter ranges extends to nearly 10 seconds in time and $2 \mathrm{~km}$ in depth - loses it's coherence from multiple forward scattering through interaction with internal waves and mesoscale energetics. Because refraction is adequate to explain the scattering physics [15], the ray limit should suffice for understanding long-range propagation. Some manifestations of the underlying chaotic dynamics should be observed.

It has been suggested that there exists a "predictability horizon" at the range of propagation defined by the scale over which chaotic dynamics develops 16. Beyond this range, the wave fields should appear as random superpositions of many plane waves [17,18 which would imply that acoustic field intensity fluctuations are Rayleigh distributed 19:20]. Several problems crop up beyond the predictability horizon. It becomes increasingly difficult to get numerically calculated rays to converge to true rays of the system. Worse, semiclassical approximations (i.e. wave front reconstructions) from the rays might fail for fundamental reasons related to the breakdown of stationary phase approximations, but one should recognize that more optimistic viewpoints exist on this issue 21 23. Whether or not this is true, it is currently not known to what extent tomographic inversions fail for any system beyond its predictability horizon where eigenrays are proliferating exponentially fast with increasing range. In order to begin addressing these and related issues, we focus on the 'forward propagation problem' by performing a statistical analysis that should be much less 
sensitive to the difficulties engendered by the predictability horizon.

In fact, justifications for statistical laws derived by invoking stochastic or ergodic postulates are often ultimately founded on the presence of fully developed chaos; see for example Ref. 24]. Systems that once were approached by stochastic methods have more recently begun to be regarded from the perspective of dynamical systems. The two approaches mostly give consistent results, but there are important distinctions. Stochastic ray modeling is the traditional approach to the geometric limit of the problem of WPRM [25]. This nondeterministic treatment leads one to pessimistic conclusions regarding the overall stability expected in an ocean acoustic pulse at sufficiently long range 16. By carefully defining the Lyapunov exponent, it turns out to be roughly half the value reported in Ref. [16]. The scales relevant to the ocean are such that this factor two increase in an important length scale might prove to be significant. Also, for this problem, the validity of the stochastic or ergodic assumptions deserves to be critically examined. It is not obvious that a dynamical systems perspective would lead to similar pessimistic conclusions as does the stochastic ray theory. For example, the predictability horizon concept that has grown out of the chaotic dynamics point of view does not necessarily lead to a sudden transition - regular behavior at short ranges, completely stochastic just beyond - and remnants of stability that violate assumptions of stochasticity could persist well into the horizon's initial onset. We anticipate several features of deterministic dynamics playing an interconnected role in this regard, but we focus on the importance of only one, intermittent-like dynamics. Intermittency is a common feature for nonintegrable dynamical systems [26,27]. For the ray acoustics problem, intermittent-like behavior is evident through the appearance of rays which persist in remaining relatively insensitive to their initial conditions (also environment) for remarkably long ranges, as measured on the inverse scale of the mean Lyapunov exponent. It might then be expected that the existence of intermittent-like dynamics might allow linear based tomographic inversions based on acoustic ray models to be suitable to greater ranges than previously anticipated.

One objective of this article is to illustrate the existence of intermittent-like dynamics in the generic longrange ocean acoustics problem. This is a direct consequence of the wide variability in the eigenvalues of the stability matrix which is defined in Sect. IIIA. We demonstrate that the magnitude of its largest eigenvalue follows a lognormal distribution, and that the stability exponent follows a Gaussian distribution. Importantly, there is preliminary evidence suggesting that these distributions are robust, i.e. that they would be found in much more realistic, sophisticated ocean models [28]. To be more explicit, if one knows the probability density of the stability exponents, then one can determine the expected measure of intermittent-like rays that will persist out to the reception range. It follows that these rays will not re- quire extremely precise numerical interpolation schemes for quantities such as the gradient of the potential.

The model upon which we rely in this paper is admittedly extremely simplified. However, it is not the model that is of concern, it is whether or not general features of simplified WPRM models carry over to the ocean itself. If we are careful enough, the simplifications that we accept remove non-essential complications for uncovering the general physical features of interest, and no more. A follow-up study to this one is underway which uses a more realistic ocean sound speed model. It is important for confirming the applicability of our results to long-range ocean acoustics experiments.

The organization is as follows: in Sect. II, we introduce and motivate a simple model leading to a one-degreeof-freedom, non-autonomous Hamiltonian dynamical system for the rays. This is followed by a discussion of the analysis methods which are most critical for our study. They are based on the stability matrix and its well known properties. Sect. IV examines the fluctuation behavior of the stability exponents giving their densities as a function of range. The proportion of intermittent-like rays is deduced and compared with the numerical results of the model. We finish with a discussion and conclusions.

\section{FROM WAVE EQUATION TO RAY MODEL}

We briefly outline the assumptions and approximations leading to the highly idealized ray model used in this paper. The primary physics we are concerned about involve refraction of acoustic energy due to volume inhomogeneities in the ocean sound speed. We assume that interactions of the acoustic energy with both the surface and sub-bottom are negligible. For multi-megameter ranges of propagation in mid-latitude, deep ocean environments, a significant amount of acoustic energy is received that satisfies this assumption [3]. As alluded to above, the necessary assumptions leading to the primary results are that: i) the linear, one-way Helmholtz wave equation is valid (the important point here is that backscattering is negligible), and ii) the spatial scales of the sound speed field are long compared to the acoustic wavelength so that ray theory is justified. A detailed derivation is readily available 29]. We point out a priori that the coordinate system is three-dimensional Cartesian $\mathbf{x}=(r, y, z)$, with $r$ the range from the source, $y$ the transverse or cross-range coordinate, and $z$ the depth from the surface. Thus, Earth curvature effects are neglected.

The fundamental starting point is the linear acoustic wave equation [7]:

$$
\frac{1}{c^{2}} \frac{\partial^{2} \psi(\mathbf{x} ; t)}{\partial t^{2}}=\nabla^{2} \psi(\mathbf{x} ; t)
$$

where $\psi(\mathbf{x} ; t)$ is the complex scalar wave function whose real part denotes the acoustic pressure. The sound speed field $c$ can be taken as a function of space $\mathbf{x}$ only whereby 
it has been assumed that the time scales for the propagation of the acoustic wave function are small compared to the time scale associated with the evolution of the sound speed field. For non-dispersive sources, the acoustic group and phase speeds are equivalent, and one can linearly transform Eq. (1) from time to frequency, arriving at a Helmholtz equation with the magnitude of the wave vector defined as $k=2 \pi f / c$, where $f$ is the continuous wave $(\mathrm{CW})$ source frequency. Attenuation effects can of course be incorporated by modifying $k$ to be a complex quantity, but since we are interested in: 1) acoustic energy that interacts negligibly with the ocean bottom, and 2) typical sources operating at frequencies with minimal volume attenuation (with center frequencies of about $100 \mathrm{~Hz}$ [6]), ignoring attenuation effects seems reasonable. Also, one can similarly derive a reduced wave equation which includes variations in density, but we ignore this effect because it is known to be important predominantly with acoustic energy that interacts with the ocean sub-bottom, which is not considered herein.

The next assumption (which is quite a reasonable one) is that the strength of the sound speed fluctuations, whatever the physical process that produces them, are small. This allows one to neglect backscattered acoustic energy, and admits the one-way Helmholtz wave equation, whereby one assumes a primary direction of propagation along the range. The so-called "standard parabolic approximation" is invoked next. This allows one to derive a linear partial differential wave equation of parabolic type for the complex envelope of $\psi$. The principle assumption is that this envelope wave function evolves slowly on the scale of the acoustic wavelength. There many flavors of parabolic approximations that have varying degrees of phase errors in the complex wave function $\psi$ as compared to the one-way Helmholtz equation [30], but we choose to use the standard parabolic approximation, which takes the form

$$
-\frac{i}{k_{0}} \frac{\partial \phi(y, z ; r)}{\partial r}=\frac{1}{k_{0}^{2}} \nabla_{\perp}^{2} \phi(y, z ; r)+V(y, z ; r) \phi(y, z ; r),
$$

where the transverse Laplacian is represented by $\nabla_{\perp}^{2}=$ $\partial_{y}^{2}+\partial_{z}^{2}$, and the variable $r$ is the range (propagation variable), but plays an exact analogous role to time in the Schrödinger equation of quantum mechanics. The parameter $k_{0}=2 \pi f / c_{0}$ represents the reference wave number, and depends on the choice of a reference sound speed $c_{0}$, which we take to be $1.5 \mathrm{~km} / \mathrm{s}$. The potential, $V(y, z ; r)$, is related to the sound speed fluctuations as

$$
V(y, z ; r)=\frac{1}{2}\left[\left(\frac{c_{0}}{c(y, z ; r)}\right)^{2}-1\right] \sim \frac{\delta c(y, z ; r)}{c_{0}},
$$

where the sound speed variations away from an average profile has been expressed as $c(y, z ; r)=c_{0}+\delta c(y, z ; r)$. The sound speed fluctuations refract the rays and lead to chaos in a deterministic, mathematically defined sense. Under the parabolic approximation, the basic problem maps precisely onto problems of quantum chaos [31]. The fields of long range acoustic propagation in the ocean and quantum chaos thus have the opportunity of crossfertilization.

Because the instability does not critically depend on having multiple degrees of freedom, we make a significant, practical simplification in the model of ignoring the depth degree of freedom $(z)$; see Ref. [16] for a more detailed discussion of the model presented here. The system could be thought of as lying in the plane of the sound channel axis, but this is really just the generic problem of WPRM (see, for example, [2]). The gain in simplicity more than compensates for the loss of realism at this point as long as the main physical phenomena carry over to more realistic models. As was mentioned in the Introduction, preliminary evidence for our main results have been found in recent calculations incorporating a much more realistic model 28].

The magnitude of the wave vector $k$ is large enough that for the purposes of this study, we can focus on the ray limit. The rays can be generated by a system of Hamilton's equations

$$
\begin{aligned}
& \frac{d y}{d r}=\frac{\partial H(y, p ; r)}{\partial p}, \\
& \frac{d p}{d r}=-\frac{\partial H(y, p ; r)}{\partial y},
\end{aligned}
$$

where $y$ and $p$ are the phase space variables cross-range (position) and horizontal slowness (momentum) respectively. The independent variable $r$ denotes range. Correspondence with Eq. (2) necessitates that the Hamiltonian is explicitly

$$
H=\frac{p^{2}}{2}+V(y ; r) .
$$

The physical meaning of momentum is $p=\tan \theta$, where $\theta$ represents the angle a ray subtends in cross-range about the range axis.

The state of the ocean is constantly changing, and its exact state is unknown. A statistical ansatz is thus fruitful for making assertions concerning its "typical" state. Assuming isotropy in the sound speed fluctuations in range and cross-range, the potential is taken to be a realization of a zero-mean, stationary, random function. Thus a single correlation length scale $L$ exists. The standard deviation is denoted by $\epsilon=c_{0}^{-1}\left\langle\delta c^{2}\right\rangle^{1 / 2}$, where $\left\langle\delta c^{2}\right\rangle^{1 / 2}$ is the root-mean-square fluctuation of the sound speed about $c_{0}$. Typical values for underwater acoustics are $\epsilon=O\left(10^{-3}\right)$, and $L=O(100) \mathrm{km}$, but both $\epsilon$ and $L$ vary plus or minus an order of magnitude depending on what ocean structure is considered and the geographic location. For purposes of studying a fully defined, deterministic dynamical system, we complete the description of $V$ by defining its correlation function to be Gaussian, 


$$
\langle V(y ; r) V(y+\delta y ; r+\delta r)\rangle=\epsilon^{2} \exp \left[-\left(\delta y^{2}+\delta r^{2}\right) / L^{2}\right]
$$

We exploit this single scale throughout the rest of this article by transforming space variables as $r \rightarrow r / L$ and $y \rightarrow y / L$, so the physical dimensions will always be in units of $L$. One should envision the potential as being deterministic, even though it is selected from an ensemble of realizations. This implies that the potential is to be considered a highly complicated (albeit smooth and fixed) function of both $y$ and $r$. To provide some idea of the character of this potential, contours of sound speed fluctuations based on a typical region of $V(y ; r)$ is shown contoured in Fig. 11. The boundary conditions are taken as open in $y$, but numerically $y$ is treated as periodic, with the ray coordinate unfolded a posteriori to simulate the open boundary condition. A variety of initial conditions are possible with the restriction that the initial momentum is always kept small enough that the parabolic approximation is valid all along the rays. The rays deriving from two such initial conditions are plotted in Fig. 2 which shows their phase space portraits (position, momentum). In the absence of a varying potential, the solutions to the equations of motion are $p(t)=p_{0}$, $q(t)=p_{0} t+q_{0}$. In this figure, rays would trace out vertical lines except in the case, $p_{0}=0$, in which rays would show up as points, $(q(t), p(t))=\left(q_{0}, 0\right)$. With the potential included, the rays trace out a random-walk-like motion with some drift as they move further away from the $p=0$ line.

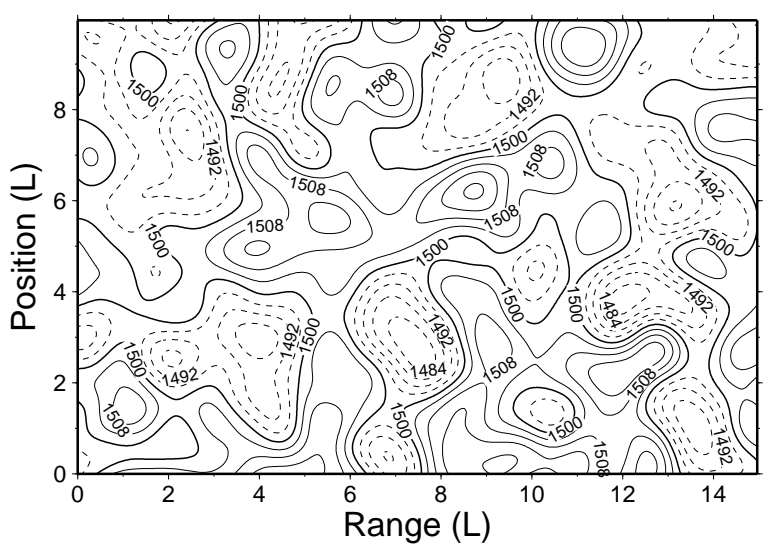

FIG. 1. A portion of a realization of $V(y ; r)$ whose full domain is $20 \mathrm{~L} \times 320 \mathrm{~L}$ in $y$ and $r$ respectively. The realization is constructed by the method described in Ref. 16. Contours are labeled in units of sound speed $(\mathrm{m} / \mathrm{s})$. The heavy solid contour line indicates the reference sound speed of $1500 \mathrm{~m} / \mathrm{s}$. The normalized root-mean-square fluctuations is $\epsilon=5 \times 10^{-3}$.

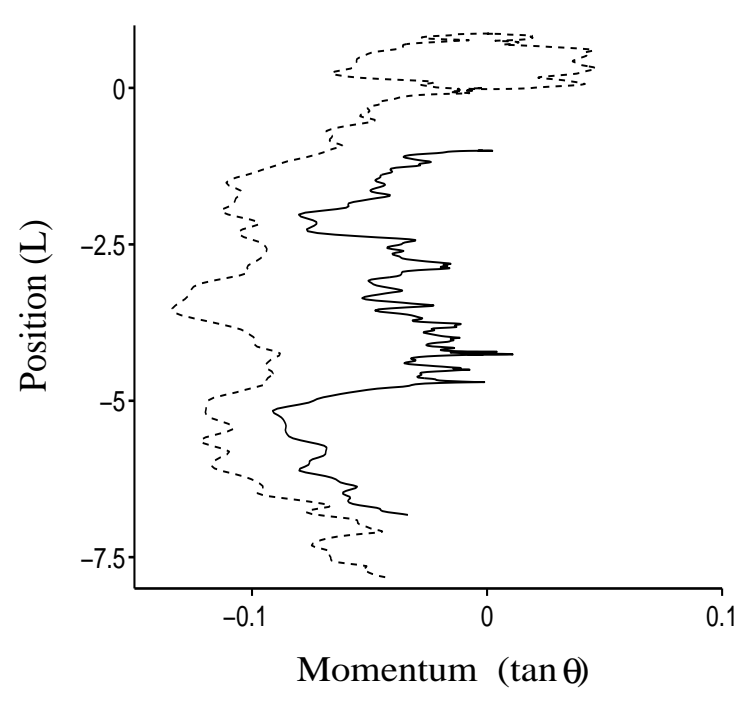

FIG. 2. Two distinct ray trajectories which have travelled through the sound speed field characterized in Fig. 11 are shown out to the range of $160 \mathrm{~L}$. The trajectory which starts at zero position and zero momentum (dashed line) is highly unstable, and the trajectory which starts at unit position and zero momentum (solid line) is stable.

\section{ANALYSIS METHODS}

The standard analysis of ray stability in the theory of dynamical systems begins with the stability matrix. From here, it is possible to calculate whether a ray is stable or unstable, what its Lyapunov exponent is, and for the unstable ray, determine the orientations of the associated stable and unstable manifolds that characterize the exponential sensitivity to initial conditions. All of our results and conclusions are based on the behavior of the stability matrices of the rays in the model introduced in the previous section. The stability matrix is a strictly local analysis in range about some particular reference trajectory. It may be stable at one range, yet for a greater range be unstable. There is no restriction that various portions of its full history cannot have completely different stability properties. In fact, one expects the portions to be almost entirely uncorrelated [24].

Often research done in chaotic dynamics uses either time (range) independent or periodic Hamiltonians, and the stability matrix is investigated about periodic orbits. The Hamiltonian of Eq. (5) is aperiodic, and as many others have done before, we slightly generalize those treatments by considering arbitrary, aperiodic rays.

\section{A. Stability Matrix}

The stability matrix for a ray describes the behavior of other rays that remain within its infinitesimal neighborhood, $\{\delta y, \delta p\}$, for all ranges. It is derived by linearizing 
the dynamics locally; see Ref. [32 for more details. At the range $r$, one has

$$
\left(\begin{array}{l}
\delta p_{r} \\
\delta y_{r}
\end{array}\right)=M\left(\begin{array}{l}
\delta p_{0} \\
\delta y_{0}
\end{array}\right)
$$

with the stability matrix being given by the partial derivatives

$$
M=\left(\begin{array}{ll}
m_{11} & m_{12} \\
m_{21} & m_{22}
\end{array}\right)=\left(\begin{array}{ll}
\left.\frac{\partial p_{r}}{\partial p_{0}}\right|_{y_{0}} & \left.\frac{\partial p_{r}}{\partial y_{0}}\right|_{p_{0}} \\
\left.\frac{\partial y_{r}}{\partial p_{0}}\right|_{y_{0}} & \left.\frac{\partial y_{r}}{\partial y_{0}}\right|_{p_{0}}
\end{array}\right) .
$$

The multi-dimensional generalizations are immediate. The $m_{21}$ matrix element is well known for its appearance in the prefactor of the standard time (range) Green's function of the parabolic equation; it therefore gives directly information on wave amplitudes.

The evolution of $M$ is governed by

$$
\frac{d}{d r} M=K M,
$$

with the initial condition $M(r=0)$ being the identity matrix, and

$$
K=\left(\begin{array}{cc}
-\frac{\partial^{2} H}{\partial y \partial p} & -\frac{\partial^{2} H}{\partial y^{2}} \\
\frac{\partial^{2} H}{\partial p^{2}} & \frac{\partial^{2} H}{\partial y \partial p}
\end{array}\right) \Longrightarrow\left(\begin{array}{cc}
0 & -\frac{\partial^{2} V}{\partial y^{2}} \\
1 & 0
\end{array}\right)
$$

The latter form is the simplification relevant for Hamiltonians of the so-called mechanical type as in Eq. (5). Since Eq. (9) represents linear, coupled, first-order differential equations, the elements of $M$ can be numerically calculated as a function of range simultaneously with the calculation of its reference ray using identical numerical techniques, e.g. variable step, fourth-order Runge-Kutta.

\section{B. Stability and Lyapunov exponents}

The stability matrix has several important properties. It can be viewed as generating a linear, canonical transformation, and therefore its determinant is equal to unity. It is diagonalized by a linear, similarity transformation

$$
\Lambda=L M L^{-1} \Longrightarrow\left(\begin{array}{cc}
\lambda & 0 \\
0 & \lambda^{-1}
\end{array}\right)
$$

where the last form applies specifically to the case of a single degree of freedom. Here, the second eigenvalue must be the inverse of the first in order for $\operatorname{det}[M]=1$. The diagonalizing similarity transformation leaves the sum of the diagonal elements (trace), $\operatorname{Tr}(M)$, invariant. It is then clear that $\operatorname{Tr}(M)$ is real, and three distinct cases may arise. The first is $|\operatorname{Tr}(M)|<2$ which is linked to stable motion, and it is then customary to denote $\lambda=\exp (i \theta r)$. The second case is $|\operatorname{Tr}(M)|=2$, and it is often called marginally stable because it is the boundary case between stable and unstable motion. The third case represents unstable motion, and is characterized by $|\operatorname{Tr}(M)|>2$. In Fig. 2, a typically stable ray is represented by the solid line, and a typically highly unstable ray is represented by the dashed line. Their distinctions are not immediately obvious.

The evolution of neighboring rays about a ray that has $|\operatorname{Tr}(M)|<2$ satisfied from the source to the reception range will undergo only rotations in phase space, and subsets of phase space of finite measure where this behavior dominates the dynamics is precisely where intermittentlike rays reside. Fig. 3 illustrates this characteristic behavior by showing a group of stable rays winding about each other as they propagate. The dashed ray in the group is the stable ray of Fig. 2. They perform their "random walks", yet remain winding about each other. For the purposes of this paper, we make a slightly generalized definition of intermittent-like rays as being all those for which $|\operatorname{Tr}(M)|$ remains sufficiently small over the range of propagation, i.e. not far from two.

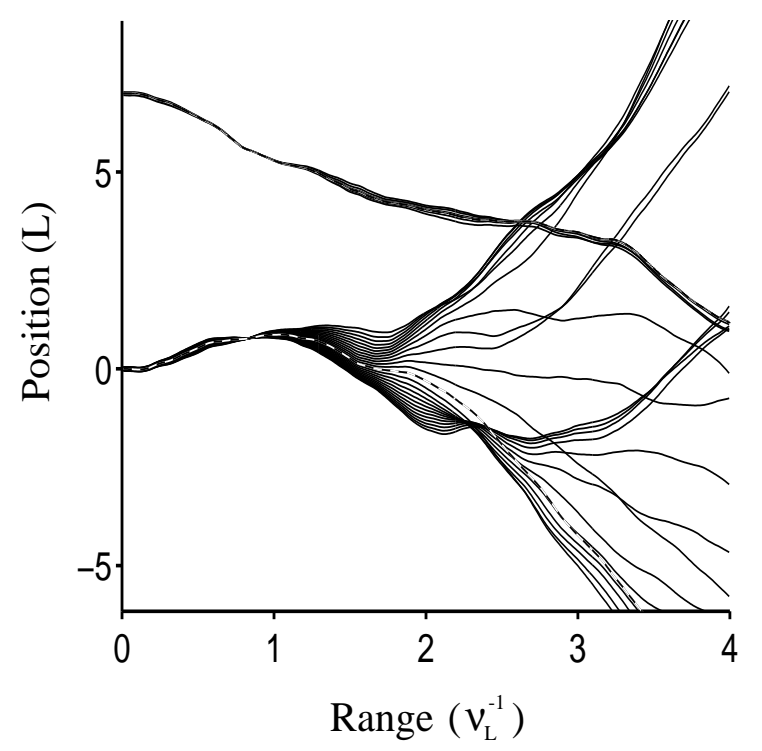

FIG. 3. Two bundles of rays surrounding the stable and unstable rays of Fig. 2 (dashed lines). For the unstable bundle, 12 rays on each side of the reference ray were chosen, each initially with zero momentum and uniformly sampling the initial position over a $0.1 \mathrm{~L}$ window about the reference ray. The stable bundle used only 2 rays on each side of the reference ray, but used the same initial condition domain as for the unstable bundle; this bundle's position is translated to $7 \mathrm{~L}$. Note the range scale is in units of the inverse Lyapunov exponent $\nu_{L}^{-1}$, defined in Eq. (12), and for the potential characterized in Fig. 1, is approximately $42.94 \mathrm{~L}$.

For unstable motion, it is customary to denote $\lambda=$ $\pm \exp (\nu r)$ where $\nu$ is positive and real. The neighboring rays move hyperbolically relative to each other. We add a collection of unstable (chaotic) rays onto Fig. 3 to illustrate the distinction between neighboring groups of stable and unstable rays. The dashed ray is the highly 
unstable ray from Fig. 2. The rays were selected to span the same size initial neighborhood as the stable group, yet they fan out and become completely independent. For the unstable case one can introduce a definition for the Lyapunov exponent as

$$
\nu_{L} \equiv \lim _{r \rightarrow \infty} \frac{\ln (|\operatorname{Tr}(M)|)}{r} .
$$

Note that there is no ensemble averaging implied in the definition of $\nu_{L}$. None of the theory presented thus far prevents it from taking on a distinct value for each ray for each realization of the random potential. In Sect. [V], the value of $\nu_{L}$ will be shown to be independent of the particular ray, and the particular realization of the potential as well. It thus defines a unique length scale, $\nu_{L}^{-1}$, which is used from Fig. 3 onward wherever the range variable is involved.

For unstable motion, $|\lambda|$ tends to be very large leaving $\lambda^{-1}$ negligible. With little inaccuracy, $\operatorname{Tr}(M)=\lambda+$ $\lambda^{-1} \approx \lambda$, even for finite ranges. One then deduces a stability exponent, $\nu$ from $\operatorname{Tr}(M)$ as

$$
\nu=\frac{\ln |\operatorname{Tr}(M)|}{r} \text {. }
$$

Thus $\nu$ depends on the particular ray and varies with range whereas the Lyapunov exponent has no range dependence by definition.

For any fixed range, an ensemble of $\nu$ can be created by considering various initial conditions (by exploiting the isotropic and stationary properties of $V(y ; r)$ ), and different realizations of $V(y ; r)$. The resulting statistical densities of $|\operatorname{Tr}(M)|, \rho_{|\operatorname{Tr}(M)|}(x)$, and similarly $\nu, \rho_{\nu}(x)$, are the main objects of concern; the two densities are directly tied to each other. The cumulative probability distribution is given as

$$
F_{\nu}(x)=\int_{-\infty}^{x} \mathrm{~d} x^{\prime} \rho_{\nu}\left(x^{\prime}\right)
$$

which provides a useful tool for numerically studying the behavior of $\rho_{\nu}(x)$. It also has the utility of directly giving the proportion of nearly stable rays up to some argument set to a maximum instability criterium, $\nu=x$.

We denote the mean and variance respectively as

$$
\begin{aligned}
& \nu_{0}=\langle\nu\rangle=\int \mathrm{d} x x \rho_{\nu}(x), \\
& \sigma_{\nu}^{2}=\left\langle\left(\nu-\nu_{0}\right)^{2}\right\rangle=\int \mathrm{d} x\left(x-\nu_{0}\right)^{2} \rho_{\nu}(x),
\end{aligned}
$$

where the brackets \langle\rangle denote ensemble averaging. For any real $\gamma$, ensemble averages of powers of $|\operatorname{Tr}(m)|$ are expressed as

$$
\left\langle|\operatorname{Tr}(M)|^{\gamma}\right\rangle=\langle\exp (\gamma \nu r)\rangle=\int \mathrm{d} x \exp (\gamma x r) \rho_{\nu}(x) .
$$

Note that the case of $\gamma=-1 / 2$ relates to wave amplitude statistics resulting from a semiclassical reconstruction of the wave field, and will be discussed in a future work.
To continue the theoretical development, it is useful to introduce a slightly modified stability exponent, $\bar{\nu}$ :

$$
\bar{\nu} \equiv \frac{\ln \left\langle|\operatorname{Tr}(M)|^{2}\right\rangle}{2 r} .
$$

Clearly $\bar{\nu}$ is necessarily greater than $\nu_{0}$ because of the important distinction of ensemble averaging before taking the natural logarithm as opposed to the inverse order and the root mean square fluctuation contributions. It is shown in the next section that the Lyapunov exponent becomes $\nu_{L}=\lim _{r \rightarrow \infty} \nu_{0}$, and not $\lim _{r \rightarrow \infty} \bar{\nu}$ which surprisingly remains greater than $\nu_{L}$. Near a parameter regime motivated by the ocean, we find numerically that analytical estimates of $\nu_{L}$ as being equal to $\bar{\nu}$ are roughly double their actual values.

\section{Stochastic analysis results}

An analytic estimate of $\bar{\nu}$ can be derived from previous analytic results based on a stochastic analysis which involves a strong Markovian assumption [33,34,16]. It was verified in Ref. 16] that, in the context of the present acoustic ray model, the stochastic analysis predictions for $\bar{\nu}$ (actually $\nu^{\prime}$, see text ahead) matched to a high degree of precision with numerical tests. In fact, no statistically significant deviations were observed. Thus, although the stochastic system is not strictly mathematically equivalent to the deterministic dynamics, we accept the applicability of those specific results at sufficiently long ranges (defining this range scale is admittedly not as trivial to determine for the general ocean acoustics scenario as it is for the idealized problem). We begin with

$$
\left\langle[\operatorname{Tr}(M)]^{2}\right\rangle=\left\langle m_{11}^{2}\right\rangle+\left\langle m_{22}^{2}\right\rangle+2\left\langle m_{11} m_{22}\right\rangle .
$$

By appealing to stochastic integration techniques 35 37. it has been shown that in the small- $\epsilon$, large- $r$ limit that 33, 34,16

$$
\left\langle m_{22}^{2}\right\rangle=\frac{1}{3} \exp \left(2 \nu^{\prime} r\right)
$$

where

$$
\begin{aligned}
& \nu^{\prime} \approx\left(\frac { 1 } { 2 } \int _ { 0 } ^ { \infty } d \xi \left\langle\left.\frac{\partial^{2} V(y ; r-\xi)}{\partial y^{2}}\right|_{\substack{y=y_{0} \\
p=p_{0}}} .\right.\right. \\
&\left.\left.\left.\frac{\partial^{2} V(y ; r)}{\partial y^{2}}\right|_{\substack{y=y_{0} \\
p=p_{0}}}\right\rangle\right)^{1 / 3} \\
&=(3 \sqrt{\pi})^{1 / 3} \epsilon^{2 / 3}
\end{aligned}
$$

(in dimensional units $\left.\nu^{\prime}=(3 \sqrt{\pi})^{1 / 3} \epsilon^{2 / 3} / L\right)$. The last result of Eq. (20) is for the specific example of a Gaussian single scale potential of Eq. (6). The first result of Eq. (20) is more general, but requires numerical confirmation for models with greater realism, and will also 
depend on the ray's initial conditions for models with a nonuniform background sound speed field.

By symmetry considerations of the stochastic equations, $\left\langle m_{11}^{2}\right\rangle=\left\langle m_{22}^{2}\right\rangle$. It is also deduced that $\left\langle m_{11} m_{22}\right\rangle$ can, at most, grow on the same scale. Defining a correlation coefficient,

$$
\mu=\frac{\left\langle m_{11} m_{22}\right\rangle}{\left\langle m_{22}^{2}\right\rangle}
$$

where $|\mu| \leq 1$, it follows that

$$
\left\langle[\operatorname{Tr}(M)]^{2}\right\rangle=\frac{2}{3}(1+\mu) \exp \left(2 \nu^{\prime} r\right) .
$$

Then, by using the definition of Eq. (17), one obtains

$$
\bar{\nu}=\nu^{\prime}+\frac{1}{2 r} \ln \left[\frac{2}{3}(1+\mu)\right] .
$$

Note that the second term disappears if $\mu$ equals $1 / 2$; i.e. $\left\langle m_{11} m_{22}\right\rangle=\left\langle m_{22}^{2}\right\rangle / 2$. We give its value numerically in the next section. In that case, $\bar{\nu}=\nu^{\prime}$ at finite range, and we have an analytic estimate for $\bar{\nu}$ (which has not been derived previously to our knowledge). It is also worth remarking that $\nu^{\prime}$ is not the Lyapunov exponent itself (just as $\bar{\nu}$ is not), but rather only an upper bound. By analogy with the behavior of $\bar{\nu}$ stated at the end of the last subsection, $\nu^{\prime}$ will turn out numerically to be about double the actual $\nu_{L}$.

\section{FLUCTUATIONS}

The ocean is not infinite in extent, and so the distribution of the stability exponents, $\nu$ (or $|\operatorname{Tr}(M)|$ ) at a specified range $r$, is more directly relevant to the ocean acoustics problem than the Lyapunov exponent, $\nu_{L}\left(\right.$ or $\left.\exp \left(\nu_{L} r\right)\right)$. In order to visualize the magnitude of the fluctuations we are discussing, Fig. A displays the $\ln |\operatorname{Tr}(M)|$ for eight of the rays from Fig. 3 as a function of range out to $7.5 \nu_{L}^{-1}$. By the right end of the figure, for any fixed range one ray might have a $|\operatorname{Tr}(M)|=\mathrm{e}^{13}$, and another one might have $|\operatorname{Tr}(M)|=\mathrm{e}^{0}$. At $7.5 \nu_{L}^{-1}$, there exist fluctuations in the stabilities of at least six orders of magnitude which is characteristic of broad tailed densities.

To characterize the fluctuations more quantitatively, we consider the cumulative densities for $\nu$ and $|\operatorname{Tr}(M)|$. An initial working hypothesis might be to check whether at some long, fixed range, a diagonal element of $M$, say $m_{i i}$, is distributed as a Gaussian random variable across the ensemble of $V(y ; r)$ and derive the implied cumulative densities from there. However, there ought to be an identifiable mechanism for a central limit theorem (CLT) to be operating with respect to $m_{i i}$. From Eq. (9), one can deduce that $M$ can be decomposed into a product of shorter range stability matrices. For very long $r$, consider a range $\Delta r$ which is short compared to the final range $\mathrm{r}$, yet long compared with $\nu_{L}^{-1}$. Let $N \Delta r=r$ where $N$ is large. Then it follows that the stability matrix is given by the left-ordered product

$$
M=\prod_{l=1}^{N} M_{l}
$$

where $M_{l}$ is the stability matrix for the range $l \Delta r$ to range $(l-1) \Delta r$. To a great degree of accuracy the set of $M_{l}$ should behave independently with the only correlations being amongst the matrix elements necessary for maintaining unit determinant. The stability matrix should have the statistical properties of an ensemble of products of uncorrelated, random matrices.

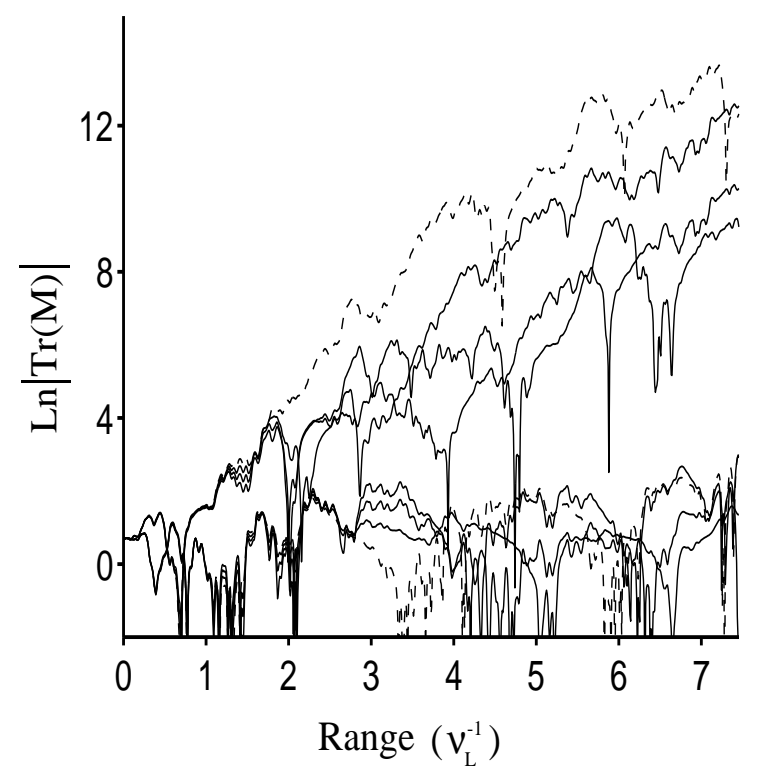

FIG. 4. The stability, $\ln |\operatorname{Tr}(M)|$, for the reference rays of Fig. 2 (dashed), and 3 of their neighboring rays initially with zero momentum and initial position shifted $0.008,0.016$, and $0.024 \mathrm{~L}$ away from the reference rays.

If there exists a limiting form for a distribution at long range $r$, one would expect the same form (with different parameters, i.e. mean, variance) at $r / 2$. In other words, the limiting form would have to be invariant under the matrix multiplication process. Denoting $m_{l, i j}$ as the matrix elements of $M_{l}$, for the $N=2$ case, we have

$$
m_{11}=m_{2,11} m_{1,11}+m_{2,12} m_{1,21}
$$

If the $m_{l, i j}$ behave as independent, random Gaussian variables, then $m_{11}$ could not be Gaussian because of the product form. The applicability of a CLT results from an additive process involving random variables. Instead, we anticipate something closer to a lognormal density because the log of a product of random variables acts like a sum of random variables. It should be mentioned here that this concept has been in use in many problems involving statistical physics [24]. 
To test whether $|\operatorname{Tr}(M)|$ is lognormally distributed, we calculate 100,000 rays through 5 realizations of $V(y ; r)$ to $7.5 \nu_{L}^{-1}(320 L)$ (a reasonable upper bound for global acoustic propagation) for values of $\epsilon=2 \times 10^{-3}, 3 \times$ $10^{-3}$, and $5 \times 10^{-3}$. If $|\operatorname{Tr}(M)|$ is distributed lognormally, then $\nu$ is distributed in a Gaussian manner by definition. In Fig. 5, we plot the cumulative density for $\nu$. The corresponding analytic Gaussian form is superposed. It is impossible to distinguish the numerical results from the Gaussian form from this plot. A similar plot for $|\operatorname{Tr}(M)|$ carries little new information, and is not pictured in this paper, though we have verified its excellent consistency with a lognormal density as well. By plotting the differences between the numerical and analytical curves for three different ranges, we see in Fig. 6 that the consistency with a Gaussian density is excellent, and that as range increases the consistency of $\rho_{\nu}(x)$ with a Gaussian density improves. Note the small scale of the deviations. We have verified that they are roughly of the order of expected sample size errors for the curve at maximum range.

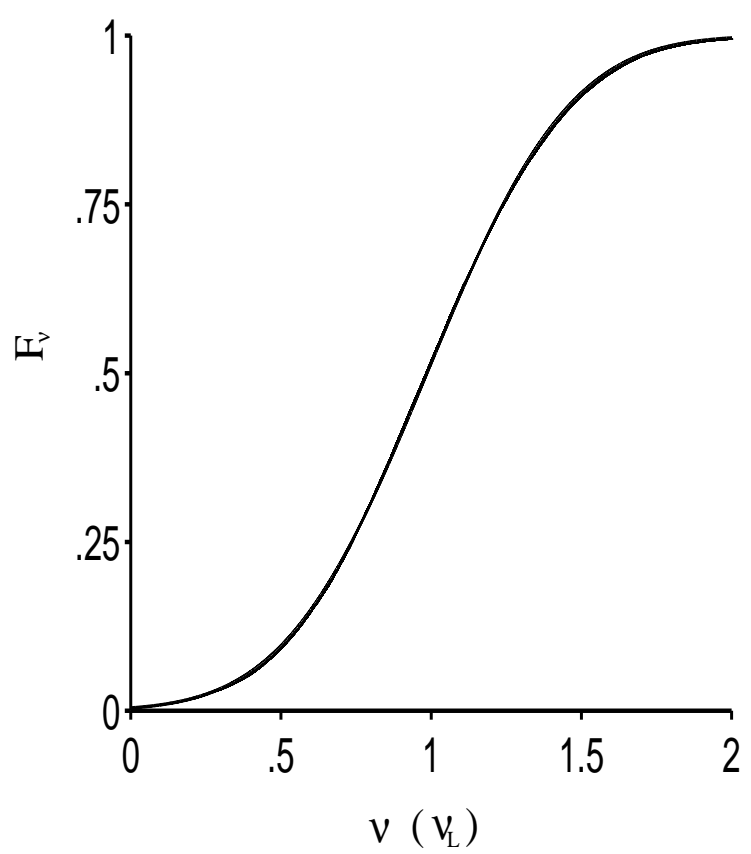

FIG. 5. Plot of the cumulative density for $\nu$ at the range of $7.5 \nu_{L}^{-1}$. The measured cumulative density is computed from 5 realizations of sound speed fields characterized in Fig. 1 $\left(\epsilon=5 \times 10^{-3}\right)$. It incorporates 20,000 rays per realization whose initial conditions uniformly sample $20 \mathrm{~L}$ in position and have zero initial momentum. Superposed is the cumulative density associated with the Gaussian density for $\nu$ [see Eq. (26)] using a value of $\nu_{0}=0.0232$ (which is our best estimate for $\nu_{L}$ derived from the same simulations) and $\bar{\nu}=\nu^{\prime}$ [see Eq. (20)]. Note the scale for $\nu$ has been normalized by $\nu_{L}$.

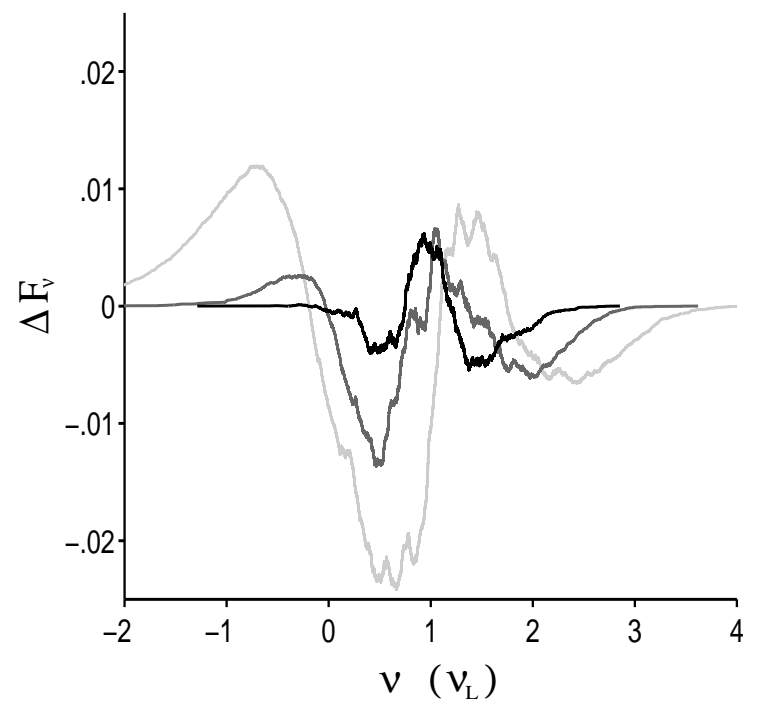

FIG. 6. Plot of difference between analytical and measured cumulative densities for $\nu$ at the ranges of 1.87 (light gray), 3.75 (medium gray), and 7.5 (black) $\nu_{L}^{-1}$. The measured cumulative densities are taken from the same simulations that produce Fig. 5. The values of the free parameters $\nu_{0}$ and $\bar{\nu}$ were adjusted within their simulated standard deviations to minimize the maximum difference for each range shown. Note the scale for $\nu$ has been normalized by $\nu_{L}$.

There are several relationships implied by the lognormal density that are straightforward to test. First, if we denote the variance of $\nu$ as $\sigma_{\nu}^{2}$, then a relationship between $\bar{\nu}$ and $\nu_{0}$ can be derived. With

$$
\begin{aligned}
\rho_{\nu}(x) & =\frac{1}{\sqrt{2 \pi \sigma_{\nu}^{2}}} \exp \left[-\frac{\left(x-\nu_{0}\right)^{2}}{2 \sigma_{\nu}^{2}}\right], \\
\bar{\nu} & =\frac{1}{2 r} \ln \left\langle e^{2 \nu r}\right\rangle \\
& =\frac{1}{2 r} \ln \left(\frac{1}{\sqrt{2 \pi \sigma_{\nu}^{2}}} \int_{-\infty}^{\infty} \mathrm{d} x \exp (2 x r) .\right. \\
& \left.\quad \exp \left[-\frac{\left(x-\nu_{0}\right)^{2}}{2 \sigma_{\nu}^{2}}\right]\right) \\
& =r \sigma_{\nu}^{2}+\nu_{0} .
\end{aligned}
$$

Inverting this last relation for $\sigma_{\nu}^{2}$, one obtains

$$
\sigma_{\nu}^{2}=\frac{\bar{\nu}-\nu_{0}}{r} .
$$

Both exponents $\bar{\nu}, \nu_{0}$ were defined (see Eq. (13, 15, 17)) to be independent of $r$ to leading order; see the upper panel of Fig. 7 where $\bar{\nu}, \nu_{0}$ are plotted as a function of range. The stochastic approximation for $\nu^{\prime}$ also given matches precisely the value of $\bar{\nu}$ implying that $\mu=1 / 2$. From numerical simulations, it turns out that $\mu$ is 0.466 , but this number is poorly determined due to sample size errors. There is no discernible $r$-dependence in either $\bar{\nu}$ or $\nu_{0}$ beyond the scale at which the stochastic approximation 
begins to work for $\bar{\nu}$. They maintain a rather constant ratio of 2.20 amongst themselves. The lognormal $|\operatorname{Tr}(M)|$ density thus implies that the standard deviation of $\rho_{\nu}(x)$ approaches zero as $r^{-1 / 2}$. Again there is excellent consistency; see the lower panel of Fig. 7 where $\sigma_{\nu}$ is plotted versus $\left[\left(\bar{\nu}-\nu_{0}\right) / r\right]^{1 / 2}$. Thus, in the limit of $r \rightarrow \infty, \rho_{\nu}(x)$ goes to a $\delta$-density; all $\nu$ converge to the single value $\nu_{0}$. This value would also have to be the Lyapunov exponent from the definition in Eq. (12), and the Lyapunov exponent would be a constant for all trajectories independent of the specific realization of the potential or the initial conditions. It appears that the approach of $\nu_{0}$ to $\nu_{L}$ is so rapid as to warrant replacing $\nu_{0}$ with $\nu_{L}$ in all the formulae of this section. In fact, the lower panel of Fig. 7 actually incorporates our best value for $\nu_{L}$ and not $\nu_{0}$ as a function of range.
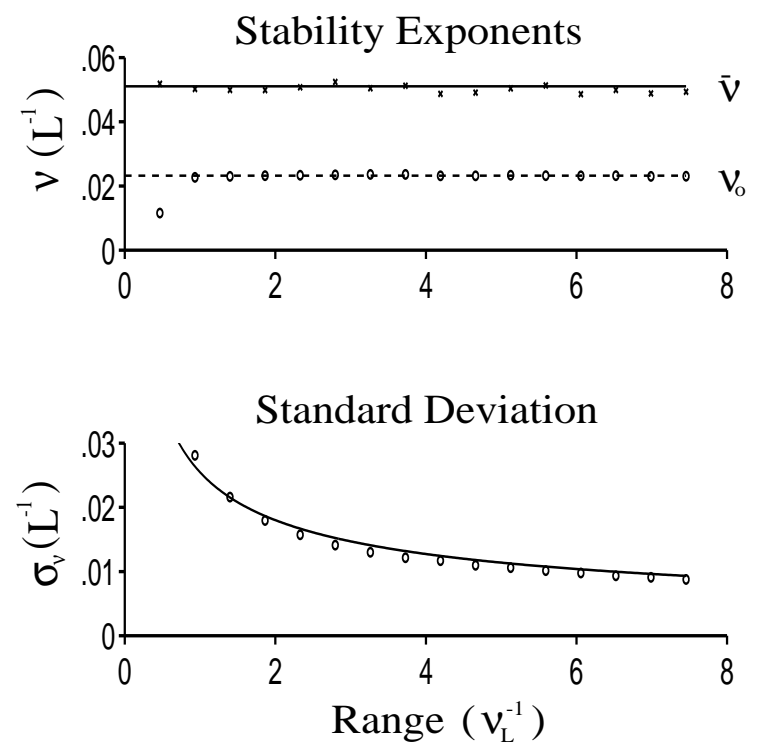

FIG. 7. Upper panel: The range dependence of $\bar{\nu}$ derived from simulations (x's) with the solid line indicating the analytical value $(=0.0510)$ determined by Eq. (20). Also shown is the range dependence of $\nu_{0}$ derived from simulations (o's) with the dashed line indicating our best estimate of $\left\langle\nu_{0}\right\rangle=0.0232$ derived by taking the mean of the $\nu_{0}$ values at ranges beyond $2 \nu_{L}^{-1}$. Lower panel: The standard deviation of $\nu$ as a function of range derived from simulations (o's), and the analytical estimate (solid line) from Eq. (27) using the value $\left\langle\nu_{0}\right\rangle$ for $\nu_{0}$ (as described in the upper panel) and Eq. (20) for $\bar{\nu}$.

A consequence of the lognormal density for $|\operatorname{Tr}(M)|$ is that the density of $|\operatorname{Tr}(M)|^{\gamma}$ for any real $\gamma$ must also be lognormally distributed. This follows from the fact that $\gamma \nu$ would be Gaussian distributed with mean $\gamma \nu_{0}$ and variance $\gamma^{2} \sigma_{\nu}^{2}$, and $\gamma \nu=\ln |\operatorname{Tr}(M)|^{\gamma} / r$. Thus $\gamma$ enters as a linear scale factor in the parameters that define the lognormal density. It is given by

$$
\rho_{|\operatorname{Tr}(M)| \gamma}(x)=\sqrt{\frac{1}{2 \pi r\left(\bar{\nu}-\nu_{0}\right)}} \frac{1}{|\gamma| x} .
$$

$$
\exp \left[\frac{-\left(\ln (x) / \gamma-\nu_{0} r\right)^{2}}{2 r\left(\bar{\nu}-\nu_{0}\right)}\right], x \geq 0 .
$$

Straightforward integration gives

$$
\left\langle|\operatorname{Tr}(M)|^{\gamma}\right\rangle=\exp \left(\left[\gamma \nu_{0}+\gamma^{2}\left(\bar{\nu}-\nu_{0}\right) / 2\right] r\right)
$$

for its ensemble averaged value. Note that the $\gamma=2$ case for which the stochastic theory was worked out is the only one independent of $\nu_{0}$, and thus also $\nu_{L}$ in the large- $r$ limit. Using Eq. (29), a variety of estimates for the Lyapunov exponent can be constructed. For example, for $r$ large enough

$$
\nu_{L} \approx \frac{2}{r} \ln \langle|\operatorname{Tr}(M)|\rangle-\frac{1}{2 r} \ln \left\langle|\operatorname{Tr}(M)|^{2}\right\rangle
$$

as given in [24]. Another example would be

$$
\nu_{L} \approx \frac{1}{r} \ln \left\langle|\operatorname{Tr}(M)|^{2}\right\rangle-\frac{1}{4 r} \ln \left\langle|\operatorname{Tr}(M)|^{4}\right\rangle
$$

etc.

Another interesting, rather curious consequence of the constant ratio of $\bar{\nu}$ to $\nu_{0}$ is that $\bar{\nu}$ does not approach $\nu_{L}$ in the $r \rightarrow \infty$ limit even though $\rho_{\nu}(x) \rightarrow \delta\left(x-\nu_{0}\right)$. Care must be taken to perform the non-commuting operations of taking the infinite range limit and ensemble averaging in the correct order. Furthermore, the variation of $|\operatorname{Tr}(M)|$ grows without bound as a function of range $r$, in spite of the fact that all the trajectories possess equal stability exponents in the limit $r \rightarrow \infty$. From Eq. (29), it follows that

$$
\sigma_{|\operatorname{Tr}(M)|}^{2}=\mathrm{e}^{\bar{\nu} r}\left(\mathrm{e}^{\bar{\nu} r}-\mathrm{e}^{\nu_{0} r}\right) \sim \mathrm{e}^{2 \bar{\nu} r}
$$

where the last form applies in the large- $r$ limit, even though $\sigma_{\nu}^{2}$ is approaching zero.

Finally, we point out that a lognormal density has long tails and, as already noted, allows for many orders of magnitude fluctuations in $|\operatorname{Tr}(M)|$. To return to the issue of intermittent-like rays, at any range, all rays whose corresponding $|\operatorname{Tr}(M)|$ are less than some $\mathrm{O}(1)$ constant can be considered as intermittent-like. Values of $e$ or $e^{2}$ could be taken as criteria, for example. The equivalent criteria expressed for the maximum of $\nu$ would be $O\left(r^{-1}\right)$. In the present model the proportion of intermittent-like rays approaches zero as $r \rightarrow \infty$, but for finite range the proportion of intermittent rays up to some maximum value $|\operatorname{Tr}(M)|=x$ is given by the cumulative density

$$
\begin{aligned}
F_{|\operatorname{Tr}(M)|}(x) & =F_{\nu}\left(\frac{\ln (x)}{r}\right) \\
& =\frac{1}{2}\left(1+\operatorname{erf}\left[\frac{\ln (x)-\nu_{0} r}{\sqrt{2 r\left(\bar{\nu}-\nu_{0}\right)}}\right]\right),
\end{aligned}
$$

where $\operatorname{erf}(z)$ is the error function of argument $z$. With the replacement of $\nu_{0}$ by $\nu_{L}$, this gives a very interesting, nontrivial connection between the Lyapunov exponent $\left(\nu_{L}\right), \bar{\nu}$, and the proportion of intermittent rays as 
a function of range. The validity of this expression is verified in Fig. 8. The behavior is just as predicted. The small deviations seen may be indicative of some slight non-lognormal behavior in the lower tail, or it may just be due to our not using best fit values of $\bar{\nu}$ and $\nu_{0}$. For long ranges, the proportion of intermittent-like rays decreases exponentially as $a_{0} r^{1 / 2} \exp \left(-b_{0} r\right)$ where $a_{0}$ and $b_{0}$ can be deduced from the asymptotic properties of the error function, and this behavior is independent of the precise criterium used for the maximum desired $|\operatorname{Tr}(M)|$. As can be seen in Fig. 8, 10\% of the initial ray density remains stable or nearly stable out to ranges of order $5 \nu_{L}^{-1}$. This $10 \%$ of the initial acoustic energy is then only linearly sensitive to the fluctuating sound speed field, and since energy remains in coherent bundles (see Fig. 3), they will be expected to have a longer time coherence over repeated experiments as the environment evolves. Performing repeated experiments and applying coherent averaging as a filter to pick up this energy, one can imagine being able to use this apportionment of the initial acoustic pulse for acoustic tomography.

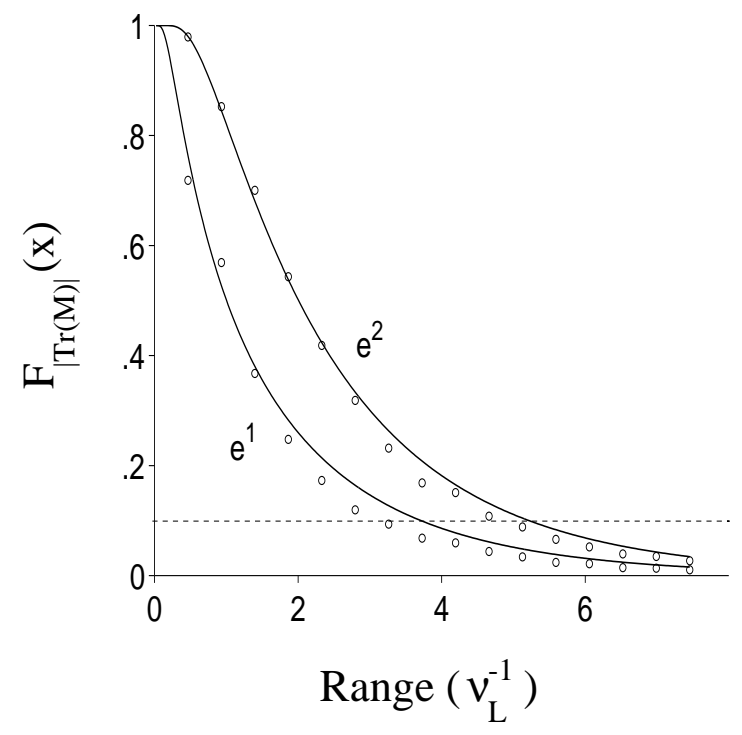

FIG. 8. The range dependence of the cumulative distribution for $|\operatorname{Tr}(M)|$ evaluated at $e^{1}$ and $e^{2}$. The measured values (o's) are derived from simulations (see caption of Fig. 5) and the theoretical curves (solid) are derived from Eq. (33) using the values of $\bar{\nu}$ and $\nu_{0}$ indicated in Fig. 0 . The horizontal dashed line at $F_{|\operatorname{Tr}(M)|}=0.1$ indicates the range at which $10 \%$ of the ray density remains nearly stable.

\section{DISCUSSION AND SUMMARY}

Long-range, low-frequency sound propagation in the ocean has been previously investigated both as a problem of wave propagation through a random medium, and as a basis for tomography. Several outstanding quandaries remain that our results only begin to address: i) in the early arriving portion of a wave front, there seems to be more coherence and stability than would be expected from an analysis based on stochastic ray techniques common to the subject of WPRM; ii) one expects that as one moves from the weak focusing to strong focusing regimes (roughly speaking, from short range to long range), there should be a transition from lognormally distributed wave field intensities to Rayleigh distributed ones. Data analyses suggest that the lognormal densities extend well beyond the weak focusing regime, and the cross-over is not understood theoretically; and iii) related to the first item, given the presence of more stability than seems consistent with theoretical modeling, how valid are the underlying assumptions of tomographic inversions performed at long ranges?

Complete solutions to these problems are well beyond the scope of this paper. Nevertheless, we believe that our results form one cornerstone for their eventual resolution. As the ocean acoustic problem mainly involves refraction, and is in a wavelength regime that should be extremely well-suited to semiclassical analysis, we have focused our attention exclusively on a simple ray model inspired by the ocean. Our approach is from a dynamical systems perspective as opposed to a stochastic ray method. It has the advantage of being a more fundamental starting point in the sense that a system's dynamics may determine where a stochastic ray method is appropriate, but a stochastic ray method just presupposes a certain randomness that may or may not actually exist in the system's dynamics.

Our main concern is the ray stability properties that govern wave field amplitudes in semiclassical approximations. A follow-up study is underway to address the phases (classical actions and geometric indices), correlations amongst ray properties, and robustness, i.e. the generality and applicability to the ocean of our results. The stability matrix is our key analysis tool because it contains all the necessary information about how stable or unstable each ray is. The distribution of stabilities reflects on statistical properties arising in the study of WPRM whereas the existence or lack thereof of stable rays impacts tomographic inversion. We also note that studying the stability matrix has the utility of providing additional strong checks on one's numerical integration techniques. Its determinant must remain unity.

We have carefully introduced several stability exponents depending upon whether ensemble averaging is taken before or after the logarithm (or at all), and whether the range is finite or the infinite range limit is taken. We have related them to the absolute value of the trace of the stability matrix which we have found to fluctuate to a high degree of consistency with a lognormal density; note that this also applies to the absolute value of individual matrix elements of the stability matrix. We have given a heuristic argument for this distribution, and are not aware of any known analytic derivations of this result.

An important consequence follows from the appearance of lognormally distributed stabilities, or equiv- 
alently Gaussian densities in the stability exponents (the logarithmic variables). As shown in Sect. IV] [see Eq. (28)], any power of the stability matrix trace, or individual matrix elements, is also distributed lognormally. Thus, each individual contribution of an eigenray to the semiclassical approximation of the Green's function has a magnitude fluctuating as a lognormal density. Further study is underway to determine theoretically the cross-over from lognormal wave field intensity distributions characteristic of the weak focusing limit to Rayleigh densities in the strong focusing limit. It is tempting to extrapolate our results to compute statistically relevant quantities such as the scintillation index (normalized variance of intensity) by using Eq. (29). Although one can immediately deduce that the normalized variance of intensity due to a single ray contribution grows exponentially with range with a e-folding scale of $\left(\bar{\nu}-\nu_{0}\right)$, one cannot infer anything about the scintillation index in the region where multipathing is important since the phase of each contributing ray must be incorporated into the calculation. Also, our work assumes one is at or beyond the regime of strong focusing. This is confirmed in the upper panel of Fig. 7, where $\nu_{0}$ is seen to converge at the range $O\left(\nu_{L}^{-1}\right)$. Since the strong Markov assumption is valid for this problem, this range can be shown to be of the order where strong focusing occurs. Thus it is erroneous to compute the scintillation index from our work in the weak focusing regime $\left(r \ll \nu_{L}^{-1}\right)$ where only one ray contributes to the intensity distribution.

All rays in the model possess identical Lyapunov exponents, and the finite-range, mean stability exponent, $\nu_{0}$, converges rapidly to it (see upper panel of Fig. 7). This follows from the finite range stability exponent acting as a Gaussian random variable with a standard deviation shrinking with range as $r^{-1 / 2}$. This is also a consequence of the lognormal density, and not the single scale nature of the sound speed fluctuations per se. This behavior may be rather general (as general as the lognormal behavior), and it would be interesting to verify it in more realistic models. It is quite unlike the $\epsilon^{2 / 3}$-scaling law for the stability exponents which should only apply to a model with a single correlation scale in range for the sound speed fluctuations.

The lognormal distribution has very broad tails. One typically observes stability matrix traces that fluctuate many orders of magnitude at a given range. Long after the appearance of highly unstable rays as a function of range, some stable or nearly stable rays will still be present. Their proportion decays essentially exponentially with range where the parameters are uniquely fixed by the Lyapunov exponent and the related stability exponent $\bar{\nu}$. However, they may be tomographically invertible, and relatively speaking, more important than their proportion would suggest. We have pointed out the distinctiveness of their behavior relative to unstable rays such as the way they twist about each other, and hang together as they propagate. Their collective properties appear to be highly correlated.
The $r^{-1 / 2}$ behavior of the standard deviation of the stability exponent leads to a paradoxical situation in which all the rays possess a unique Lyapunov exponent, yet the exponentiated quantity, the matrix elements or trace of the stability matrix possess a divergent variance as the range approaches infinity. This illustrates dramatically the differences arising when non-commuting operations, i.e. ensemble averaging, taking the logarithm, and taking the infinite range limit, are interchanged. It is the stability matrix elements which are relevant to semiclassical approximations. So the individual terms in a summation over eigenrays will vary infinitely in their relative importance.

\section{Acknowledgments}

We gratefully acknowledge helpful discussions with M. G. Brown, F. D. Tappert, J. A. Colosi, and financial support from the Office of Naval Research.

[1] J. A. Colosi, A. B. Baggeroer, T. G. Birdsall, C. Clark, B. D. Cornuelle, D. Costa, B. D. Dushaw, M. Dzieciuch, A. M. G. Forbes, B. M. Howe, D. Menemenlis, J. A. Mercer, K. Metzger, W. Munk, R. C. Spindel, P. F. Worcester, and C. Wunsch, "A review of recent results on ocean acoustic wave propagation in random media: Basin scales," IEEE Journ. of Ocean. Eng. 24, 138-155 (1999).

[2] B. J. Uscinski, "Wave Propagation and Scattering in Random Media," vol. 2, Academic Press, N.Y., (1978).

[3] W. Munk, P. Worcester,and C. Wuncsh, Ocean Acoustic Tomography (Cambridge University Press, Cambridge, 1995).

[4] A. B. Baggeroer, T. G. Birdsall, C. Clark, J. A. Colosi, B. D. Cornuelle, D. Costa, B. D. Dushaw, M. Dzieciuch, A. M. G. Forbes, C. Hill, B. M. Howe, J. Marshall, D. Menemenlis, J. A. Mercer, K. Metzger, W. Munk, R. C. Spindel, D. Stammer, P. F. Worcester, and C. Wunsch, "Ocean climate change: Comparison of acoustic tomography, satellite altimetry, and modeling," Science 281, 1327-1332 (1998).

[5] J. A. Colosi, E. K. Sheer,S. M. Flatté, B. D. Cornuelle, M. A. Dzieciuch, W. H. Munk, P. F. Worcester, B. M. Howe, J. A. Mercer, R. C. Spindel, K. M. Metzger, T. G. Birdsall, and A. B. Baggeroer, "Comparisons of measured and predicted acoustic fluctuations for a 3250$\mathrm{km}$ propagation experiment in the eastern North Pacific Ocean," J. Acoust. Soc. Am 105, 3202-3218 (1999).

[6] P. F. Worcester, B. D. Cornuelle, M. A. Dzieciuch, W. H. Munk, B. M. Munk, J. A. Mercer, R. C. Spindel, J. A. Colosi, K. Metzger, T. G. Birdsall, and A. B. Baggeroer, "A test of basin-scale acoustic thermometry using a large-aperture vertical array at $3250-\mathrm{km}$ range in the eastern North Pacific Ocean," J. Acoust. Soc. Am 105, 3185-3201 (1999). 
[7] S. M. Flatté, R. Dashen, W. H. Munk, and F. Zachariasen, Sound Transmission through a Fluctuating Ocean (Cambridge University Press, Cambridge, 1979).

[8] J. A. Colosi, S. M. Flatté, and C. Bracher, "Internal-wave effects on 1000-km oceanic acoustic pulse propagation: Simulation and comparison with experiment," J. Acoust. Soc. Am. 96, 452-468 (1994).

[9] J. L. Spiesberger, E. Terray, and K. Prada, "Successful ray modeling of acoustic multipaths over a $3000-\mathrm{km}$ section in the Pacific," J. Acoust. Soc. Am. 95, 3654-3657 (1994).

[10] V. I. Tatarskii, Wave propagation in a turbulent medium (McGraw-Hill, New York, 1961).

[11] D. R. Palmer, M. G. Brown, F. D. Tappert, and H. F. Bezdek, "Classical chaos in nonseparable wave propagation problems," Geophys. Res. Lett. 15, 569-572 (1988).

[12] K. B. Smith, M. G. Brown, and F. D. Tappert, "Ray chaos in underwater acoustics," J. Acoust. Soc. Am. 91, 1939-1949 (1992).

[13] K. B. Smith, M. G. Brown, and F. D. Tappert, "Acoustic ray chaos induced by mesoscale ocean structure," J. Acoust. Soc. Am. 91, 1950-1959 (1992).

[14] E. Ott, Chaos in Dynamical Systems (Cambridge University Press, Cambridge, 1993).

[15] J. Simmen, S. M. Flatté, and G. Wang, "Wave front folding, chaos, and diffraction for sound propagation through ocean internal waves", J. Acoust. Soc. Am. 102, 239-255 (1997).

[16] M. A. Wolfson and F. D. Tappert, "Study of horizontal multipaths and ray chaos due to ocean mesoscale structure," J. Acoust. Soc. Am. 107, 154-162 (2000).

[17] M. V. Berry, "Semi-classical mechanics in phase space: a study of Wigner's function," Phil. Trans. R. Soc. London A 287, 237-271 (1977).

[18] M. V. Berry, "Regular and irregular semiclassical wave functions," J. Phys. A: Math. Gen. 10, 2083-2091 (1977).

[19] V. I. Shishov, "Theory of wave propagation in random media," Izvestiya VUZ. Radiofizika 11, 866-875 (1968).

[20] R. Dashen, "Path integrals for waves in random media," J. Math. Phys. 20, 894-920 (1979).

[21] S. Tomsovic and E. J. Heller, "Long-time semiclassical dynamics of chaos: the stadium billiard," Phys. Rev. E 47, 282-299 (1993).

[22] M-A. Sepúlveda, S. Tomsovic, and E. J. Heller, "Semiclassical propagation: how long can it last?," Phys. Rev. Lett. 69, 402-405 (1992).

[23] M. G. Brown and M. A. Wolfson, preprint (2000).

[24] A. Crisanti, G. Paladin, and A. Vulpiani, Products of Random Matrices in Statistical Physics (Springer-Verlag, Berlin, 1993).

[25] L. A. Chernov, Wave Propagation in a Random Medium (McGraw-Hill, New York, 1960).

[26] A. B. Zisook, "Intermittency in area-preserving mappings," Phys. Rev. A. 25(4), 2289-2292 (1982).

[27] P. Bergé, Y. Pomeau, and C. Vidal, Order within Chaos (Hermann, Paris, 1984).

[28] J. A. Colosi, private communication. Calculations were performed using AET climatology measurements of mesoscale energetics, and with a Garrett-Munk internal wave spectrum.

[29] F. D. Tappert, "The parabolic approximation method," in Wave propagation and underwater acoustics, Lecture Notes in Physics, Vol. 70, eds. J. B. Keller and J. S. Papadakis, (Springer-Verlag, New York, 1977), Chap. V, pp. 224-287.

[30] F. D. Tappert and M. G. Brown, "Asymptotic phase errors in parabolic approximations to the one-way Helmholtz equation," J. Acoust. Soc. Am. 99, 1405-1413 (1996).

[31] E. J. Heller and S. Tomsovic, "Postmodern Quantum Mechanics," Physics Today 46, 38-46 (1993).

[32] E. J. Heller, "Wave packet dynamics and quantum chaology," in Chaos and quantum physics, eds. M.-J. Giannoni, A. Voros, and J. Zinn-Justin, Les Houches, Session LII, (Elsevier Science Publishers B. V. 1991), Course IX, pp. 549-663.

[33] A. N. Malakhov, S. N. Molodtsov, and A. I. Saichev, "To the hypothesis of a logarithmically normal distribution law of amplitude fluctuations of a light wave propagating in a randomly, inhomogeneous medium," Radiophys. Quantum Electron. 20, 169-176 (1977).

[34] V. A. Kulkarny, and B. S. White, "Focusing of waves in turbulent inhomogeneous media," Phys. Fluids 25, 17701784 (1982).

[35] G. C. Papanicolau and W. Kohler, "Asymptotic theory of mixing stochastic ordinary differential equations," Comm. Pure Appl. Math.27, 641-668 (1974).

[36] D. A. Dawson and G. C. Papanicolau, "A random wave process," Appl. Math. Optim.12, 97-114 (1984).

[37] R. L. Stratanovich, Topics in the theory of random noise, vol. 1, Gordon and Breach, N. Y., (1963). 